http://dx.doi.org/10.1590/0104-1428.1733

\title{
Zinc Layered Hydroxide Salts: Intercalation and Incorporation into Low-Density Polyethylene
}

\author{
Silvia Jaerger, Ademir Zimmermann, Sonia Faria Zawadzki, Fernando Wypych \\ Departamento de Química, Centro de Pesquisa em Química Aplicada - CEPESQ, \\ Universidade Federal do Paraná - UFPR \\ Sandro Campos Amico
Programa de Pós-Graduação em Engenharia de Minas, Metalúrgica e de Materiais - PPGE3M,
Escola de Engenharia, Universidade Federal do Rio Grande do Sul -UFRGS
}

\begin{abstract}
In this study, polymer composites using low-density polyethylene (LDPE) and layered hydroxide salts (LHS) were synthesized. The following compositions of LHS were obtained $\mathrm{Zn}_{5}(\mathrm{OH})_{8}\left(\mathrm{~A}^{\mathrm{n}-}\right)_{2 / \mathrm{n}} \cdot \mathrm{yH}_{2} \mathrm{O}$, where $\mathrm{A}$ was varied in order to obtain hydrophilic $\left(\mathrm{A}=\mathrm{NO}_{3}^{-}\right)$or hydrophobic $\left(\mathrm{A}=\mathrm{DDS}^{-}-\right.$dodecyl sulfate or $\mathrm{DBS}^{-}-$dodecyl benzene sulfonate). Synthesis was carried out by co-precipitation in alkaline medium and drying, being followed by characterization via Fourier-transform infrared spectroscopy, thermogravimetric analysis, X-ray diffraction and scanning electron microscopy. A variable amount of filler was then incorporated into the LDPE via extrusion, which was then injection molded to obtain specimens for evaluating tensile properties (Young's modulus, tensile strength, strain at break and toughness). For comparison, the sodium salts of the surfactants (NaDDS and NaDBS) were also used as fillers in LDPE. The X-ray diffraction results indicated that the hydrophobic LHS were exfoliated in the polymer matrix, whereas the hydrophilic LHS was only delaminated. In the LDPE composites, melting and crystallization temperatures were nearly constant, along with the crystallinity indexes. The mechanical properties were mainly varied when the organophilic LHS was used. Overall, fillers based on LHS, especially those containing hydrophobic anions, may be interesting alternatives in the production of reinforced thermoplastics.
\end{abstract}

Keywords: Layered hydroxide salts, nanocomposite, low-density polyethylene.

\section{Introduction}

Polyolefins have been massively used in the last decades due to their low cost, high chemical resistance and acceptable mechanical properties ${ }^{[1]}$. Low-density polyehylene (LDPE), for instance, is vastly used in the production of films for packaging, due to its comparatively high tensile and impact strength, or as a constituent of blends ${ }^{[2,3]}$. If even better mechanical, thermal and electrical properties could be incorporated into LDPE by adding fillers, the range of applications would grow wider, for instance in films for agriculture ${ }^{[4]}$, antimicrobial compounds $^{[5]}$, among others.

The combination of active micro- or nano-fillers with polymers, producing micro- or nano-composites, has been extensively studied lately ${ }^{[6]}$. Layered inorganic fillers are among the fillers that may be used, especially cationic exchange clay minerals of the 2:1 type, but these minerals also show some drawbacks related to their highly variable chemical composition, morphology, cation exchange capacity and impurity content.

Some synthetic layered materials appear as an alternative to surpass those disadvantages, especially the anionic exchange type. Layered double hydroxides (LDH) ${ }^{[7]}$ and layered hydroxide salts (LHS) ${ }^{[8]}$ belong to this family of compounds, and even though the former has been fairly well-studied, the latter is rarely found in the literature, especially when used as fillers in polymer composites ${ }^{[9-13]}$. For example, improvement of the thermal-oxidative degradation of LDPE and a slight increase in crystallization temperature was evidenced by Muksing et al. ${ }^{[14]}$, whereas significant improvement of the thermal-oxidative stability and remarkable reduction of the oxygen permeability of LDPE was reported by Coiai et al. ${ }^{[15]}$. Higher performance nanocomposites with respect to flame retardance and thermal stability are commonly attributed to a better dispersion state of the LDH in the LDPE matrix and the greater hindrance effect of LDH layers on the diffusion of oxygen and volatile products throughout the composite materials when they are exposed to burning or thermal degradation $^{[16]}$. With increasing LDH concentration, the heat release rate and its maximum during cone-calorimeter investigation were found to be significantly reduced, and the limited oxygen index and the dripping behavior improved ${ }^{[17]}$.

The LHS are obtained when a fraction of the sites occupied by hydroxyl ions in the Brucite $\left(\mathrm{Mg}(\mathrm{OH})_{2}\right)$ structure is replaced by water molecules or anions. For the latter, ions of the second coordination sphere of the metal are required to stabilize the electrostatic charge ${ }^{[18]}$. For instance, the zinc hydroxide nitrate, general formula $\mathrm{Zn}_{5}(\mathrm{OH})_{8}\left(\mathrm{NO}_{3}\right)_{2} \cdot 2 \mathrm{H}_{2} \mathrm{O}$, is a hydroxide salt in which the layers are comprised of zinc atoms octahedrally coordinated by hydroxyl anions and tetrahedrally coordinated to three hydroxyl anions and one water molecules, where the nitrate positioned between the layers is exchangeable ${ }^{[19]}$.

The use of intercalation reactions to insert mobile species in the interlayer space of the layered compounds 
is an important field of study since it allows modification of their original properties as a consequence of changes in their electronic density. The organic anions are of interest regarding possible intercalation reactions of the LHS because they not only expand the basal distance of the materials, allowing compatibilization with the hydrophobic polymers, but also show a plasticizer effect that may increase flexibility of the composites if an adequate filler content is used ${ }^{[20]}$.

In this context, this work focuses on the synthesis and characterization of LHS compounds intercalated with nitrate anions and organic surfactants anions like dodecyl sulfate (DDS) and dodecyl benzene sulfonate (DBS). Also, their use as reinforcing fillers for LDPE following a simple extrusion process is investigated.

\section{Experimental}

The $\mathrm{Zn}\left(\mathrm{NO}_{3}\right)_{2} \cdot 6 \mathrm{H}_{2} \mathrm{O}$, and $\mathrm{NaOH}$ reactants were supplied by Vetec. Intercalated anions were produced from two surfactants, sodium dodecyl sulfate (NaDDS) and sodium dodecyl benzene sulfonate (NaDBS), supplied by Synth. The LDPE polymer powder PB608 grade $(\mathrm{MFR}=30 \mathrm{~g} / 10 \mathrm{~min})$, kindly donated by Braskem (Brazil) and free from additives, was used as received.

The zinc hydroxide nitrate (called $\mathrm{ZHN}$ ), $\mathrm{Zn}_{5}(\mathrm{OH})_{8}\left(\mathrm{NO}_{3}\right)_{2} \cdot 2 \mathrm{H}_{2} \mathrm{O}$, was obtained via co-precipitation process under alkaline medium, at room temperature. A solution $(100 \mathrm{~mL})$ with $40 \mathrm{mmol}$ of $\mathrm{Zn}\left(\mathrm{NO}_{3}\right)_{2} \cdot 6 \mathrm{H}_{2} \mathrm{O}$ was prepared and later reacted with a $\mathrm{NaOH}$ solution $\left(1 \mathrm{~mol} . \mathrm{L}^{-1}\right)$, until $\mathrm{pH} \sim 7.0$. The obtained white precipitate was kept under stirring for $24 \mathrm{~h}$ and then washed with distilled water and centrifuged, repeating the process for up to 4 times. The product was then dried in a vacuum oven at $60^{\circ} \mathrm{C}$ for $48 \mathrm{~h}$.

The layered hydroxide salts (LHS) intercalated with the dodecyl sulfate (DDS) and the dodecyl benzene sulfonate (DBS) anions were also synthesized via direct co-precipitation process with constant $\mathrm{pH}$ at room temperature. A solution $(200 \mathrm{~mL})$ containing $64 \mathrm{mmol}$ of the NaDDS or NaDBS was prepared by the simultaneous addition of $100 \mathrm{~mL}$ of a solution containing $40 \mathrm{mmol}$ of $\mathrm{Zn}\left(\mathrm{NO}_{3}\right)_{2} \cdot 6 \mathrm{H}_{2} \mathrm{O}$, and correcting the $\mathrm{pH}$ by adding 1 mol. $\mathrm{L}^{-1}$ $\mathrm{NaOH}$ solution so that it remained close to neutrality. The obtained white precipitate was also washed/dried as described above.

Composites were produced by melting the LDPE with the fillers using a micro-extruder Haake MINILAB II operating with $160 \mathrm{bar}$, at $130^{\circ} \mathrm{C}$. The material was then injected at 320 bar into a mold cavity (at $40^{\circ} \mathrm{C}$ ) to produce ASTM D638 (type IV) samples in a HAAKE injection equipment MINIJET II. The following LHS contents were used: $0.1,0.2,0.5,1.0$ and $2.0 \%$ in relation to the polymer weight.

X-ray diffraction (XRD) analysis was carried out using a Shimadzu XRD-6000 equipment with $\mathrm{CuK} \alpha(1.5418 \AA)$ radiation, operating at $30 \mathrm{~mA}$ and $40 \mathrm{kV}$, scanning at $2^{\circ} \cdot \mathrm{min}^{-1}\left(0.02^{\circ}\right.$ steps $)$ for the synthesized solid compounds and at $0.5^{\circ} \cdot \mathrm{min}^{-1}$ for the LDPE composites. Fouriertransform infrared spectroscopy (FTIR) was performed in a Bio-Rad FTS 3500GX equipment operating under transmission mode with $\mathrm{KBr}$ disks and using 32 scans in the $400-4000 \mathrm{~cm}^{-1}$ range, with a nominal resolution of $2 \mathrm{~cm}^{-1}$. Scanning electron microscopy coupled with an energy dispersive X-ray spectrometer (EDS) was used for morphological/compositional evaluation of the compounds after gold sputtering. The equipment, JEOL (JSM-6360LV), operated at $15 \mathrm{kV}$ and only qualitative EDS data was used.

Thermogravimetric analysis (TGA) measurements were carried out using a Mettler Toledo TG/s-DTA 851E equipment. The samples were submitted to a heating program $\left(10{ }^{\circ} \mathrm{C} \cdot \mathrm{min}^{-1}\right)$ in the $25-1000{ }^{\circ} \mathrm{C}$ range, under oxygen atmosphere. Differential scanning calorimetry (DSC) analysis of the unfilled and filled polymers was done in a NETZSCH DSC 200 equipment under nitrogen atmosphere using the following running cycle: heating from room temperature to $150{ }^{\circ} \mathrm{C}$ (at $10^{\circ} \mathrm{C} \cdot \mathrm{min}^{-1}$ ), $5 \mathrm{~min}$ at $150{ }^{\circ} \mathrm{C}$, cooling to $-20^{\circ} \mathrm{C}$ (at $10^{\circ} \mathrm{C} \cdot \mathrm{min}^{-1}$ ), and heating up to $150^{\circ} \mathrm{C}$ (at $10^{\circ} \mathrm{C} \cdot \mathrm{min}^{-1}$ ).

Lastly, tensile testing was carried out in a universal testing machine Instron 5567, equipped with $1 \mathrm{kN}$ load cell. For each family of samples, ten specimens were tested at a speed of $10 \mathrm{~mm} \cdot \mathrm{min}^{-1}$ and a minimum of five results were used to calculate the mean.

\section{Results and Discussion}

The X-ray diffraction patterns of ZHN, of DDS and DBS intercalated hydroxide salts (named, LHS-DDS and LHS-DBS, respectively) and of the sodium salts of the surfactants (NaDDS and NaDBS) are shown in Figure 1. The diffraction pattern of the $\mathrm{ZHN}$ indicated the presence of a crystalline phase, with intense peaks related to the (200) and (400) planes. The basal spacing of the latter (monoclinic structure) was $9.66 \dot{\mathrm{A}}$, similar to the value reported in the literature, $9.8 \AA^{[21]}$.

The LHS-DDS and LHS-DBS samples showed only one, low-crystallinity, phase. Diffraction peaks related to the basal reflection planes in the stacking direction of the layered crystals (h00) appeared, indicating that the obtained products retained their layered structure. Besides, no peaks associated with the precursors, NaDDS e NaDBS salts (Figure 1c and 1e, respectively), could be seen in the products.

The basal spacing of the DBS and DDS intercalated hydroxide salts were $33.15 \dot{\mathrm{A}}$ and $26.38 \dot{\mathrm{A}}$, respectively. According to the literature ${ }^{[22]}$, the size of the dodecyl sulfate anion along the bigger axis is $19.3 \dot{A}$ and, adding that to the layer thickness $(4.8 \dot{\mathrm{A}})$ and the two tetrahedra $(2 \times 2.6 \dot{A})$, one can estimate the overall spacing as $29.3 \dot{A}$. The difference between the calculated and the theoretical value is likely to be due to hydration water molecules associated with the sulfate hydrophilic group and organic anion tilted orientation. This indicates that dodecyl sulfate anions interacted with the tetrahedron of the layered hydroxide salt, perpendicularly to the plane of the layers, similar to what was reported by Kandare et al. ${ }^{[23]}$ for copper hydroxy dodecyl sulfate. The basal spacing of the LHSDBS $(33.15 \dot{A})$ is also consistent with the intercalation of the dodecyl benzene sulfonate anion, because the size of the anion estimated with the software Hyperchem and 
taking into account the angle of the aliphatic chain bonded to the sulfonate benzene, is $21.8 \dot{\mathrm{A}}$, which yields an overall spacing of $31.8 \dot{\mathrm{A}}$ when considering the thickness of the layers and the two tetrahedra.

The FTIR spectrum of the ZHN (Figure 2a) showed a wide band in the $3500 \mathrm{~cm}^{-1}$ wavenumber region attributed to $\mathrm{O}-\mathrm{H}$ lattice vibrations and water molecules. The narrow band in $3575 \mathrm{~cm}^{-1}$ was attributed to the hydroxyl groups of the layered lattice, with well-defined vibration energies, and the intense band in $1384 \mathrm{~cm}^{-1}$ is characteristic of the free nitrate ion (v3 mode) ${ }^{[19]}$.

In the LHS-DDS and LHS-DBS products, the $3575 \mathrm{~cm}^{-1}$ band (for hydroxyl groups) is absent, which is an indication that at least some of the organic ions may have reacted with them. This is corroborated by the fact that there was a decrease in intensity of the nitrate ion characteristic band (at $\left.1384 \mathrm{~cm}^{-1}\right)$, suggesting a slight contamination of these materials with that ion. For the LHS-DDS (Figure 2b),

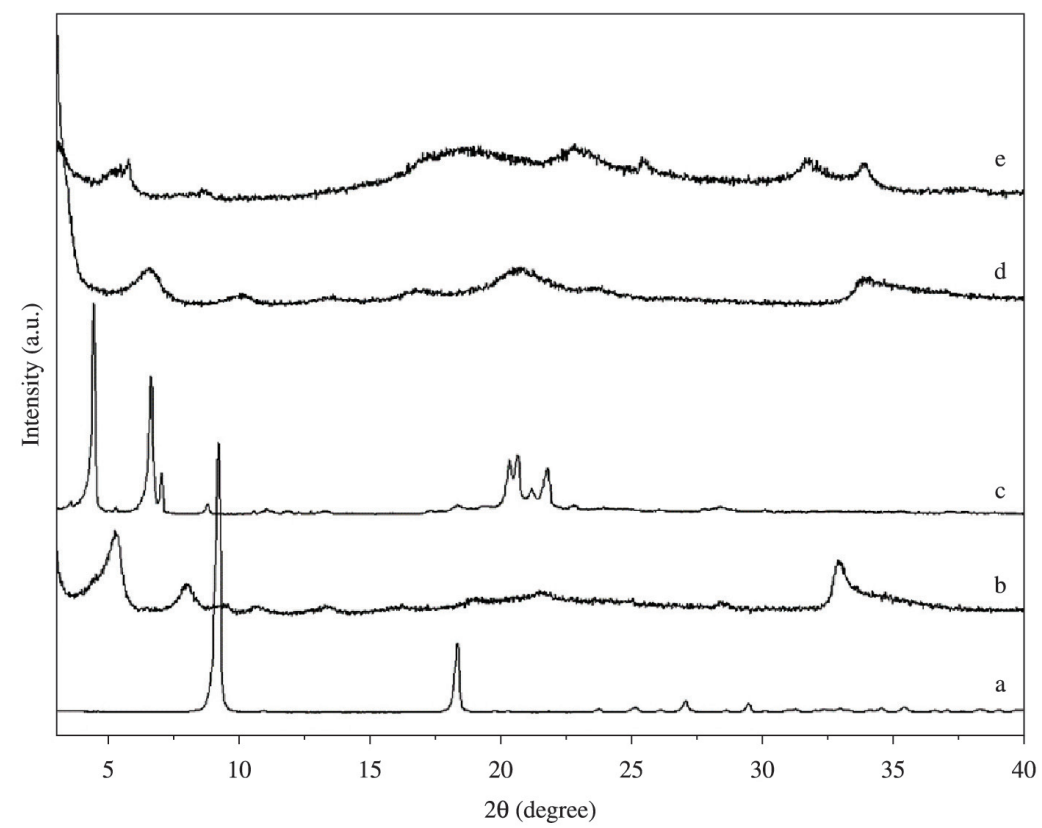

Figure 1. X Ray diffraction patterns of ZHN (a), LHS-DBS (b), NaDDS (c), LHS-DDS (d) and NaDBS (e).

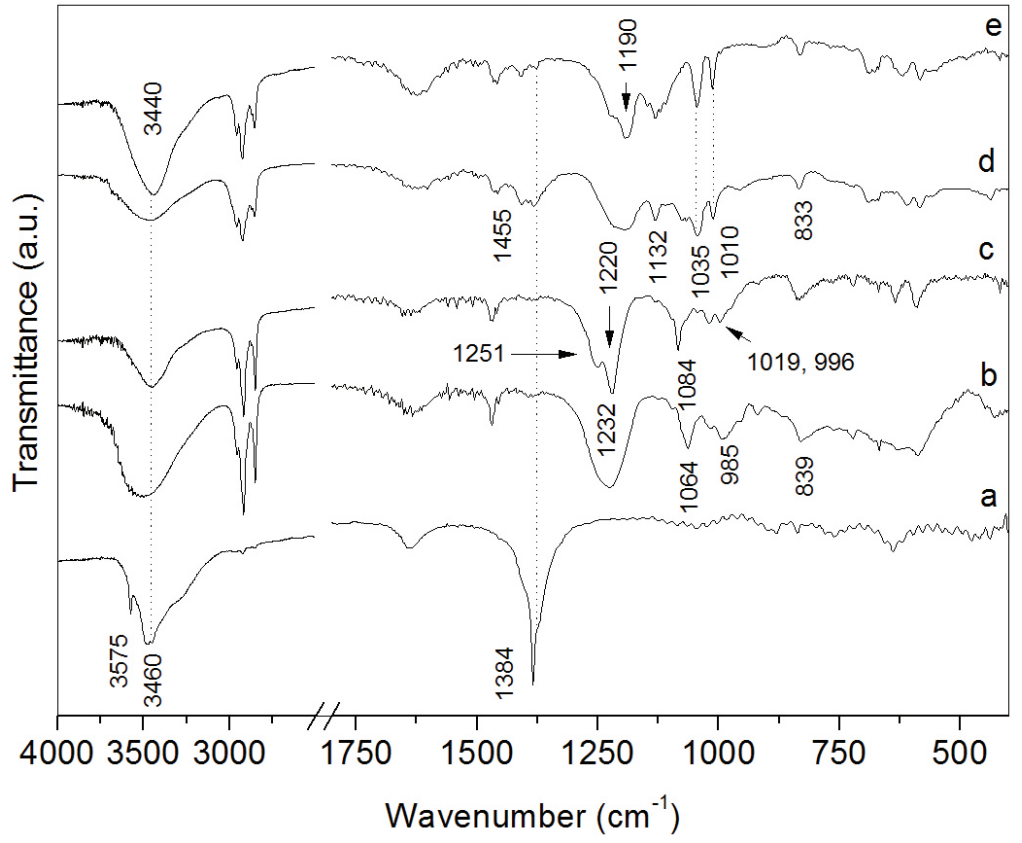

Figure 2. FTIR spectra of ZHN (a), LHS-DDS (b), NaDDS (c), LHS-DBS (d) and NaDBS (e). 
there were bands associated with the axial stretching of $\mathrm{CH}_{2}$ groups close to 2960,2923 and $2846 \mathrm{~cm}^{-1}$, and bands at $1232,1064,985$ and $839 \mathrm{~cm}^{-1}$ that represent vibration modes of the sulfate group. For the LHS-DBS, typical $\mathrm{C}-\mathrm{H}$ stretching bands at $2957 \mathrm{~cm}^{-1}\left(\mathrm{n}_{\mathrm{as}}\left(\mathrm{CH}_{3}\right)\right), 2922 \mathrm{~cm}^{-1}$ $\left(v_{a s}\left(\mathrm{CH}_{2}\right)\right)$ and $2856 \mathrm{~cm}^{-1}\left(v_{s}\left(\mathrm{CH}_{2}\right)\right)$ confirm intercalation of the organic anion (Figure 2d). The bands at 1132 and $1010 \mathrm{~cm}^{-1}$ correspond to in-plane stretching of the aromatic $\mathrm{C}-\mathrm{H}$ whereas the band at $833 \mathrm{~cm}^{-1}$ is related to out-of-plane stretching. At $1383 \mathrm{~cm}^{-1}$ and $1455 \mathrm{~cm}^{-1}$ there are bands attributed to the symmetric stretching of $\mathrm{CH}_{3}$ and flexure of $\mathrm{CH}_{2}$, respectively. In addition, bands associated with the sulfonate anions can be seen at $1129 \mathrm{~cm}^{-1}\left(\mathrm{n}_{\text {as }}(\mathrm{S}=\mathrm{O})\right)$ and $1035 \mathrm{~cm}^{-1}\left(\mathrm{n}_{\mathrm{s}}(\mathrm{S}=\mathrm{O})\right)$.

Figure $3 \mathrm{~A}-\mathrm{C}$ shows SEM images and the EDS results obtained for ZHN, LHS-DDS and LHS-DBS, respectively. For ZHN, it was possible to observe crystals with welldefined corners, similar to hexagonal plaques, which are typical of layered compounds. The product was found to contain oxygen and zinc only, as dictated by its chemical structure, being free from impurities. On the other hand, the LHS-DDS compound is finer and with distinct morphology, i.e. thinner plates of higher roughness and more rounded corners. The EDS analysis in this case indicated not only oxygen and zinc (due to the hydroxide salt) but also carbon and sulfur (due to the DDS). The LHS-DBS compound is comprised of sub-micrometric crystals and only agglomerates were seen within the SEM range (5000× magnification). Its EDS results were similar to those of the LHS-DDS.

Figure 4 shows the TGA/DTG results for the ZHN (Figure 4A) and the surfactant intercalated phases (LHS-DDS in Figure 4B, and LHS-DBS in Figure 4C). Until c.a. $140{ }^{\circ} \mathrm{C}$, two mass loss events (accounting for 5.6\%) are seen, both attributed to the loss of physisorbed or structural water. At higher temperatures, the ZHN structure starts to dehydroxylate, with the production of an intermediate phase consisting of a mixture of $\mathrm{Zn}_{3}(\mathrm{OH})_{4}\left(\mathrm{NO}_{3}\right)_{2}$ and $\mathrm{ZnO}$, which later (around $300{ }^{\circ} \mathrm{C}$ ) decomposes into $\mathrm{ZnO}, \mathrm{NO}_{2}, \mathrm{NO}$ and $\mathrm{O}_{2}{ }^{[24]}$. The overall mass loss is $32.88 \%$, consistent with the decomposition of $\mathrm{Zn}_{5}(\mathrm{OH})_{8}\left(\mathrm{NO}_{3}\right)_{2} \cdot 2 \mathrm{H}_{2} \mathrm{O}$ (theoretical mass loss of $34.69 \%$ ).

For the LHS intercalated with DDS anion (Figure 4B), the TGA/DTG results indicate a mass loss event of $3.15 \%$ within $35-113{ }^{\circ} \mathrm{C}$, which can be attributed to the loss of water. Within $113-500{ }^{\circ} \mathrm{C}$, there is a mass loss of $42.96 \%$ related to the decomposition of organic compounds, possibly the dodecyl sulfate anion ${ }^{[20]}$. In the $750-900{ }^{\circ} \mathrm{C}$ range, the production of $\mathrm{ZnO}$ and $\mathrm{Zn}_{3} \mathrm{O}\left(\mathrm{SO}_{4}\right)_{2}$ is expected, which later (at $900{ }^{\circ} \mathrm{C}$ ) decompose into $\mathrm{ZnO}, \mathrm{SO}_{2}$ and $\mathrm{O}_{2}$. This final mass loss represented $13.65 \%$, and the $\mathrm{ZnO}$ residue was of $40.24 \%$, which is consistent with that compound. For the LHS intercalated with DBS anion, (Figure 4C), the TGA/DTG results were similar. The mass loss events are c.a. $12.5 \%$ (up to $221{ }^{\circ} \mathrm{C}$ ), $24.72 \%$ (within $250-650^{\circ} \mathrm{C}$ ) and $62.78 \%$ (for higher temperatures),
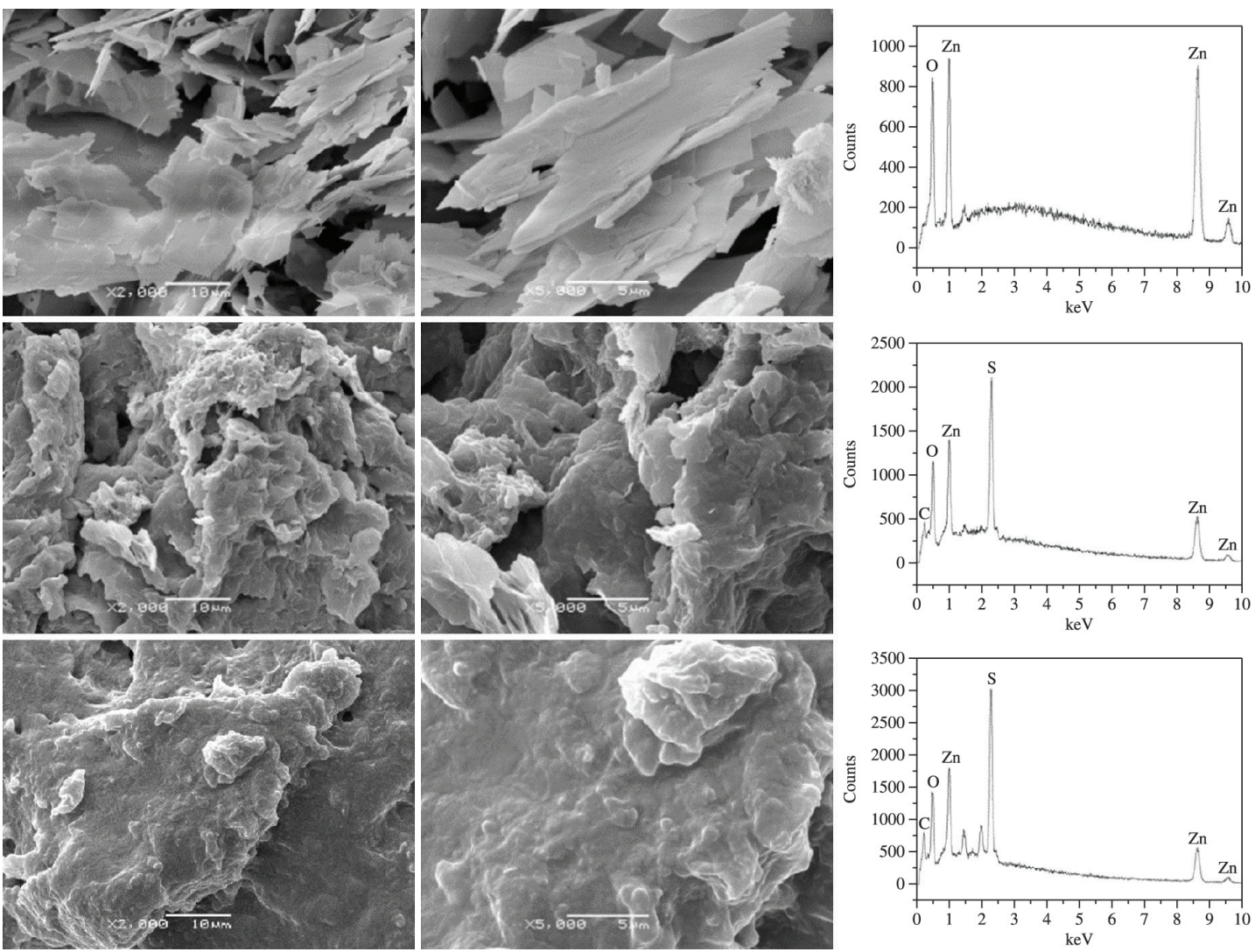

Figure 3. SEM images of ZHN (A), LHD-DDS (B) and LHS-DBS (C), with the respective EDS spectra. 
respectively. Based on theoretical calculations, the composition of the layered hydroxide salts may be estimated as $\mathrm{Zn}_{5}(\mathrm{OH})_{8}\left(\mathrm{NO}_{3}\right)_{2} \cdot 1.64 \mathrm{H}_{2} \mathrm{O}, \mathrm{Zn}_{5}(\mathrm{OH})_{8}(\mathrm{DDS})_{2} \cdot 1.77 \mathrm{H}_{2} \mathrm{O}$ and $\mathrm{Zn}_{5}(\mathrm{OH})_{8}(\mathrm{DBS})_{2} .8 .85 \mathrm{H}_{2} \mathrm{O}$ for the $\mathrm{ZHN}$, the LHS-DDS and the LHS-DBS, respectively.

The X-ray results of the ZHN/LDPE composites (Figure 5) clearly show diffraction peaks attributed to the orthorhombic crystallographic planes (110) and (200) of the polyethylene ${ }^{[25]}$, and also reflection peaks (200) and (400) of the ZHN (Figure 5A) that grow in intensity for higher filler content. The ZHN structure was preserved, which was expected due to the weak interaction between the polymeric matrix and the hydrophilic filler.

X-ray diffraction patterns of the LDPE composites with LHS-DDS and LHS-DBS are showed in Figures 5B and $5 \mathrm{C}$, respectively. In general, diffraction peaks related to the layered materials were not seen, suggesting exfoliation of the layered crystals in the composites. However, for the composite with $2.0 \%$ of LHS-DDS (Figure $5 \mathrm{C}$ ), there is a low intensity diffraction peak in the low angle region,
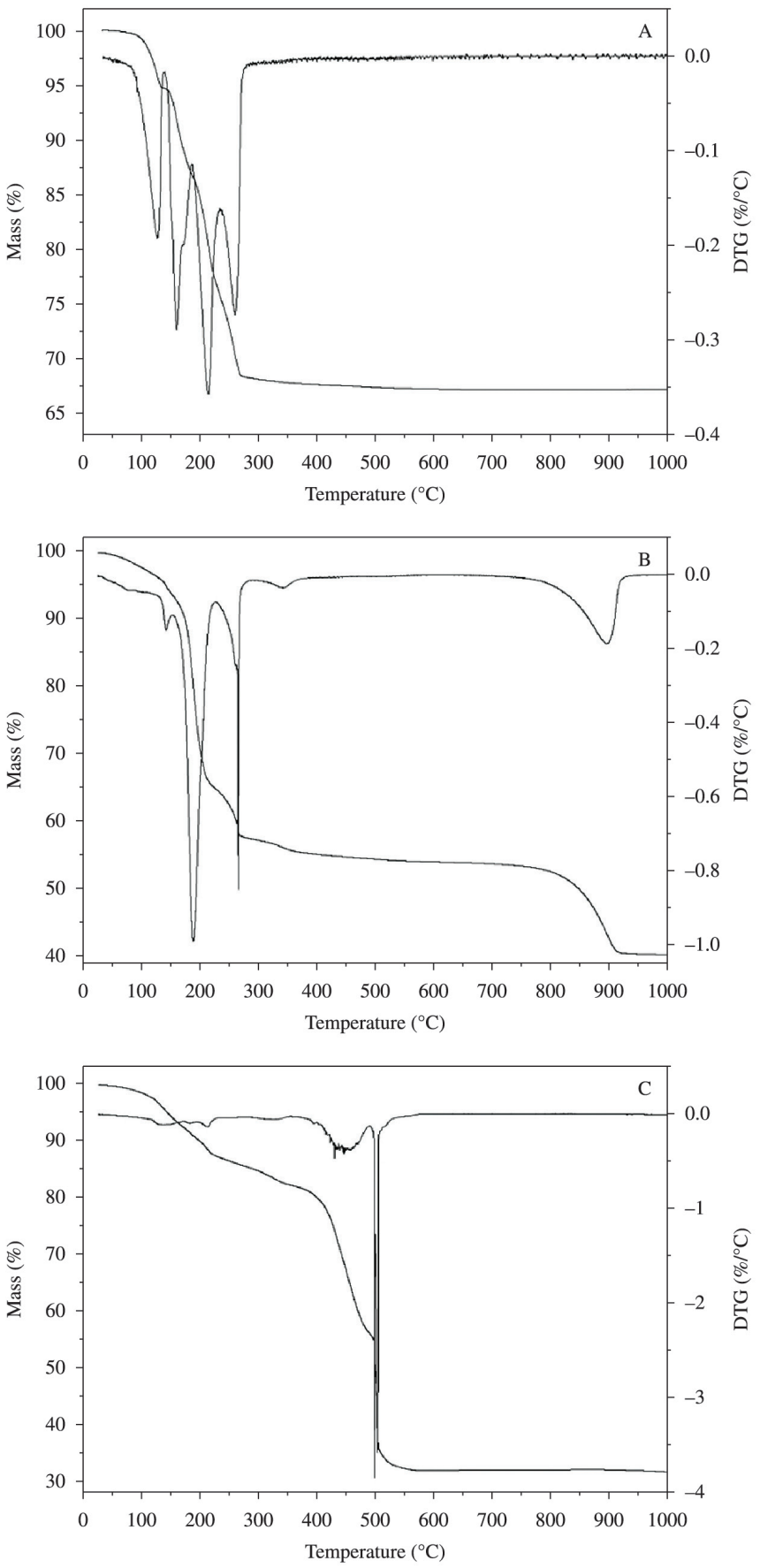

Figure 4. Thermal analysis curves (TGA/DTG) of ZHN (A), LHS-DDS (B) and LHS-DBS (C). 
around $2 \theta=3^{\circ}$. Therefore, as expected, the hydrophilic filler showed poorer exfoliation in the hydrophobic polymer structure compared to the hydrophobic LHS, differently from the findings of Youssef et al. ${ }^{[26]}$, although the later carried out "in situ" emulsion polymerization of styrene in the presence of the LDH suspended crystals.

Table 1 shows the DSC results for the pure polymer and the various LDPE composites. The melting and crystallization temperatures were little affected by the addition of the fillers. Nevertheless, the crystallization enthalpy showed certain increase especially for the composites with $0.1 \%$ and $2.0 \%$ of ZHN. For the LHS-DBS composites, crystallinity was found to decrease with the filler content. In all, the most crystalline composite was the one with $0.1 \%$ of LHS-DBS, with a change in melting and crystallization enthalpies of 2.3 and $4.4 \mathrm{~J} / \mathrm{g}$, respectively.
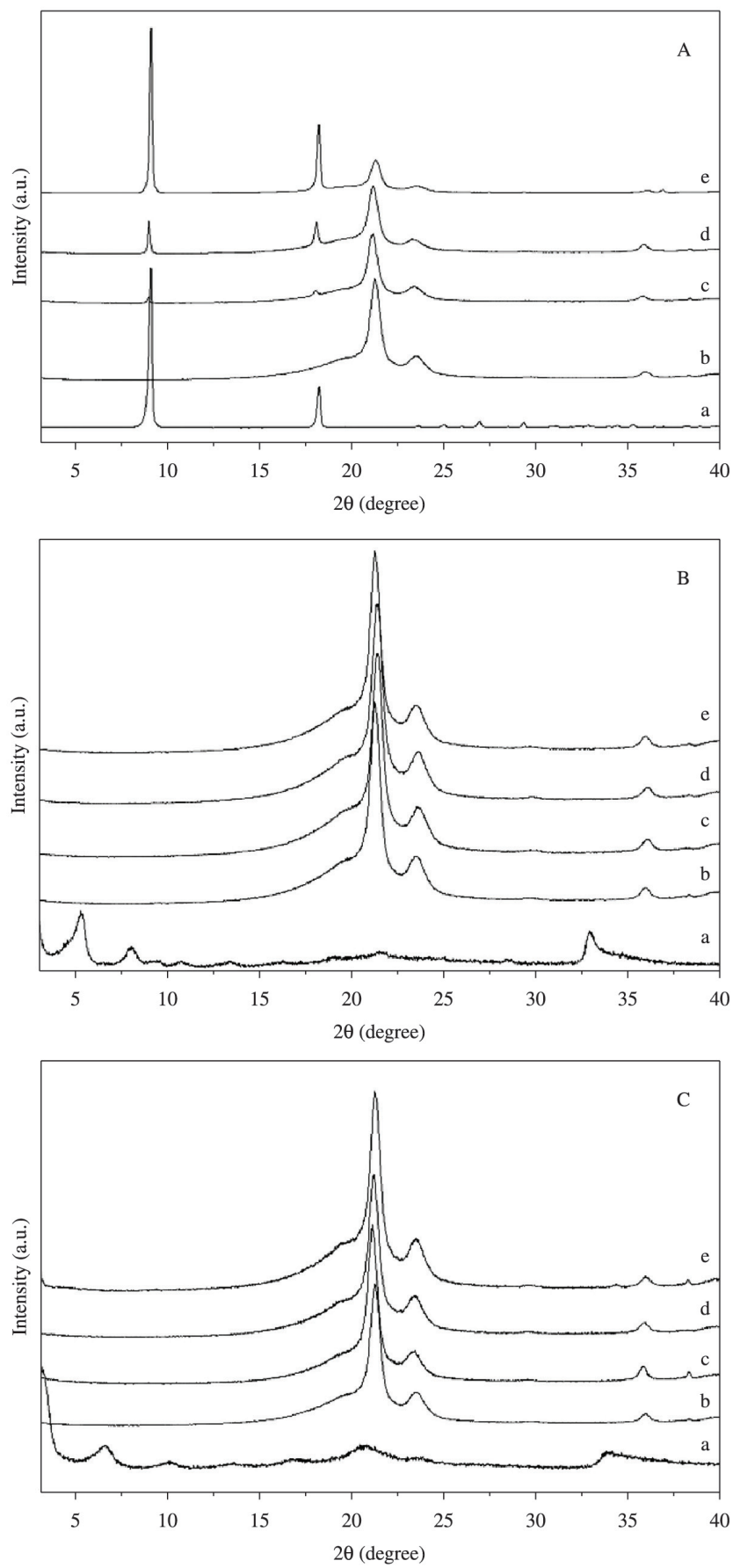

Figure 5. X-ray diffraction patterns. (A): ZHN (a), LDPE (b) and after mixing $0.5 \%$ (c), $1 \%$ (d) and 2\% (e) of ZHN with LDPE. (B): LHS-DBS (a), LDPE (b) and after mixing $0.5 \%$ (c), $1 \%$ (d) and $2 \%$ (e) of LHS-DBS with LDPE. (C): LHS-DDS (a), LDPE (b) and after mixing $0.5 \%$ (c), $1 \%$ (d) and $2 \%$ (e) of LHS-DDS with LDPE. 
Figures 6-9 shows the results of mechanical properties for the LDPE composites. Figure 6 shows that the addition of filler decreased Young's modulus of the material, but small increments were observed when surfactants of the sodium salts were used, since they allow better interaction with the polymeric chains. For the ZHN, modulus first decreased then increased when the filler content reached $2 \%$. ZHN is a hydrophilic layered material with poor interaction with the polymer, but due to its relatively large particle size, it is able to increase stiffness of the polymer, especially at higher content. It is important to bear in mind that $\mathrm{ZHN}$ retained the basal spacing nearly constant as previously shown by X-ray diffraction especially for the compounds with $1.0 \%$ and $2.0 \%$ of $\mathrm{ZHN}$, and this is coherent with these modulus results.

Modulus decreased for the compounds with DDS and DBS anions intercalated hydroxide salts regardless of the content. These hydroxide salts should show greater affinity with the matrix in comparison with the $\mathrm{ZHN}$ compounds due to the more hydrophobic character of the former, but this effect was only observed for the intermediate content levels.

The ultimate tensile strength results for the studied materials are shown in Figure 7. Addition of the DDS and DBS surfactants salts increased this property in comparison with the pure LDPE, probably due to their hydrophobic character. On the other hand, addition of small amount of ZHN and LHS-DDS decreased ultimate strength values, recovering later the original strength for larger filler amount. The lowest strength values were found for the LHS-DBS samples.

The ultimate strain results for the studied materials are shown in Figure 8. For the LHS-DDS samples, strain first increased than decreased for higher filler content, with a maximum at $0.1-0.2 \%$. However, addition of the LHS-DBS and the surfactant itself (DBS) increased strain for filler

Table 1. DSC results for the pure LDPE and the various composites.

\begin{tabular}{lccccc}
\hline \multicolumn{1}{c}{ Sample } & Filler content & $\mathbf{T}_{\mathbf{m}}\left({ }^{\circ} \mathbf{C}\right)$ & $\Delta \mathbf{H}_{\mathbf{m}}(\mathbf{J} / \mathbf{g})$ & $\mathbf{T}_{\mathbf{c}}\left({ }^{\circ} \mathbf{C}\right)$ & $\Delta \mathbf{H}_{\mathbf{c}}(\mathbf{J} / \mathbf{g})$ \\
\hline Pure LDPE & - & 104.9 & -79.2 & 88.5 & 71.3 \\
LDPE/ZHN & $0.1 \%$ & 104.3 & -93.7 & 89.6 & 76.9 \\
& $0.5 \%$ & 104.2 & -75.9 & 89.5 & 71.3 \\
LDPE/LHS-DDS & $2.0 \%$ & 105.4 & -80.4 & 87.9 & 74.1 \\
& $0.1 \%$ & 104.4 & -72.9 & 89.3 & 68.9 \\
LDPE/LHS-DBS & $0.5 \%$ & 104.1 & -86.7 & 89.9 & 73.3 \\
& $2.0 \%$ & 104.5 & -72.2 & 89.6 & 73.8 \\
& $0.1 \%$ & 104.4 & -81.5 & 89.2 & 75.7 \\
& $0.5 \%$ & 105.1 & -81.2 & 88.4 & 71.0 \\
\hline
\end{tabular}

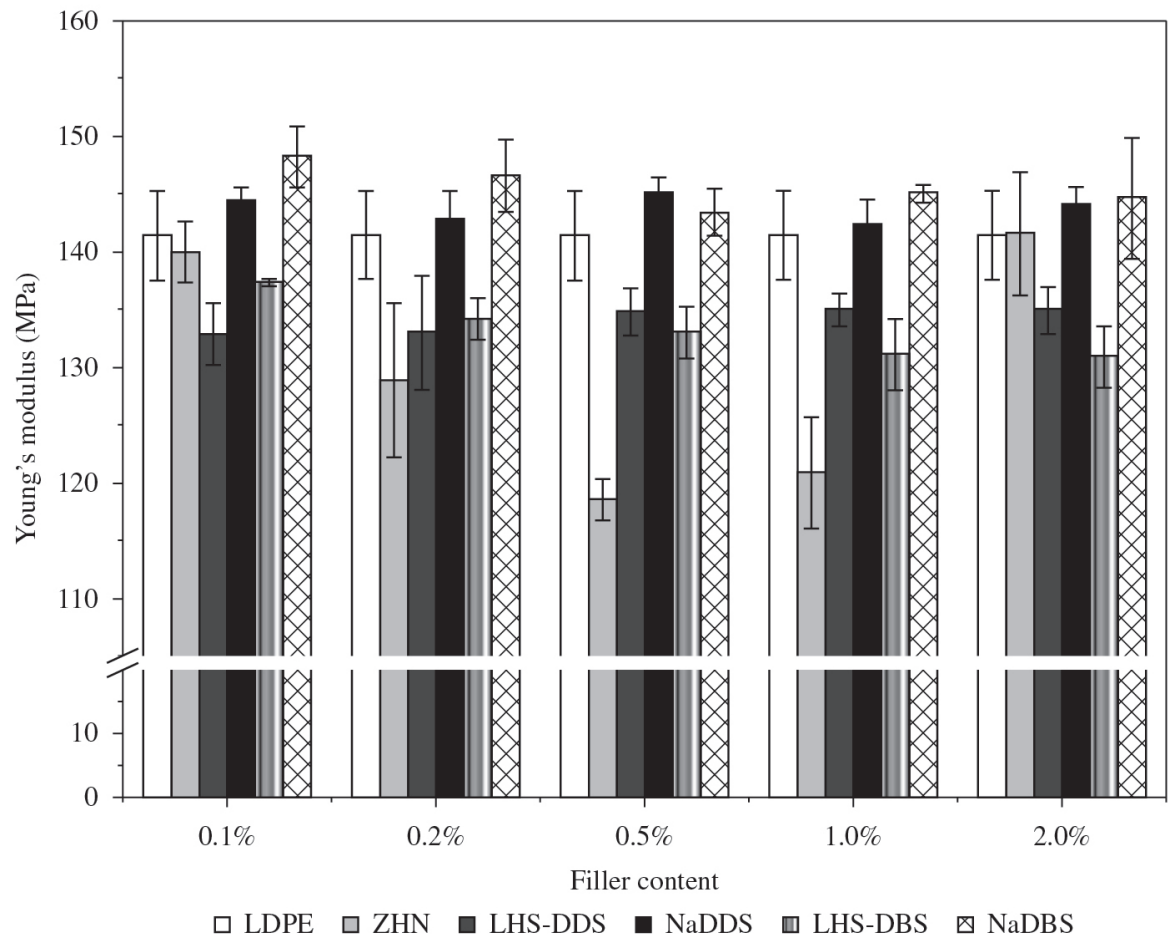

Figure 6. Young's modulus of the LDPE composites using different fillers. 
contents higher than $0.1 \%$, being even higher than that of the ZHN samples.

The composites with higher modulus tended to show lower strain at break. The affinity between the surfactant intercalated hydroxide salts and the polymer may promote yielding of the LDPE leading to higher ductility, which is beneficial in situations where the material may undergo impact events. In general, it may be said that the exfoliated fillers were able to improve strain at break of the composites perhaps acting as an internal lubricator additive, allowing

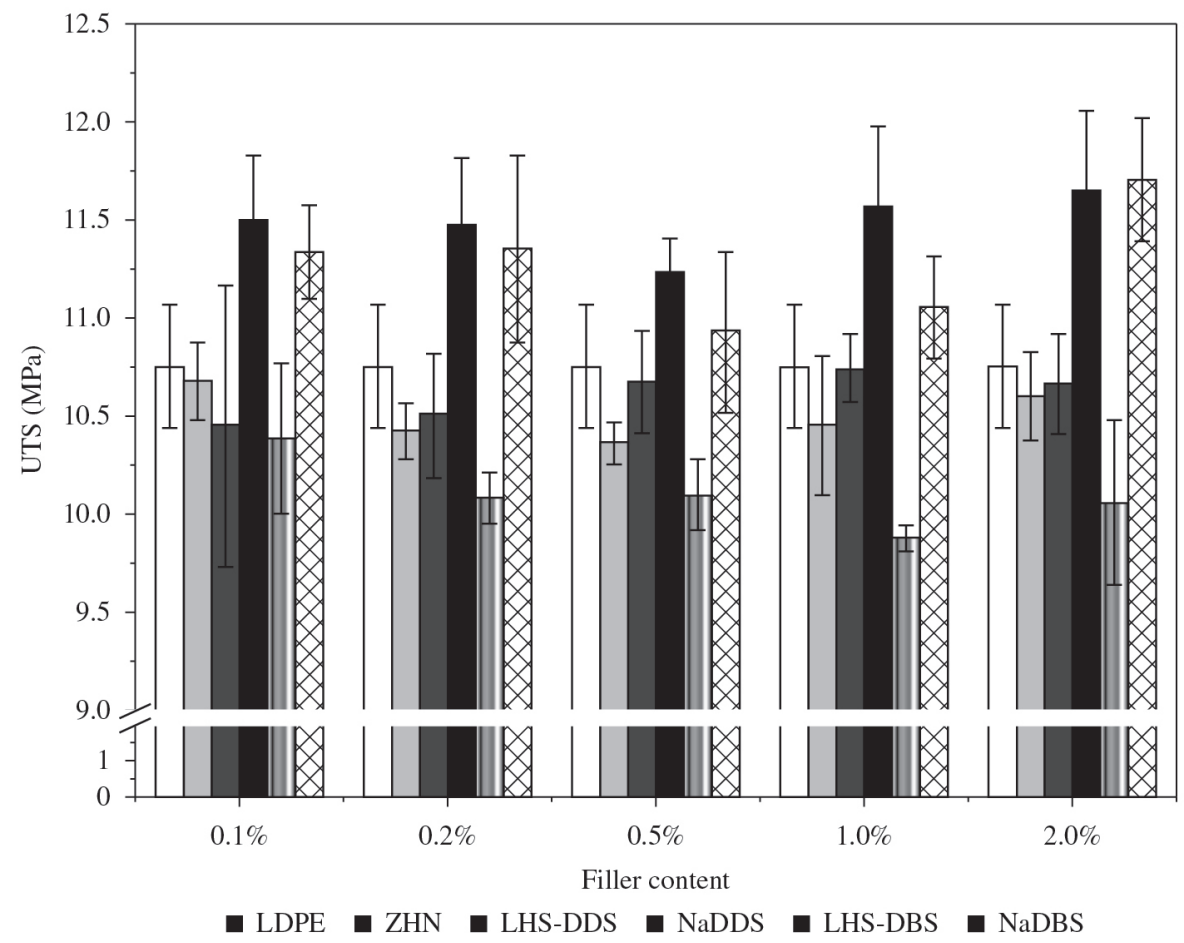

Figure 7. Tensile strength of the LDPE composites using different fillers.

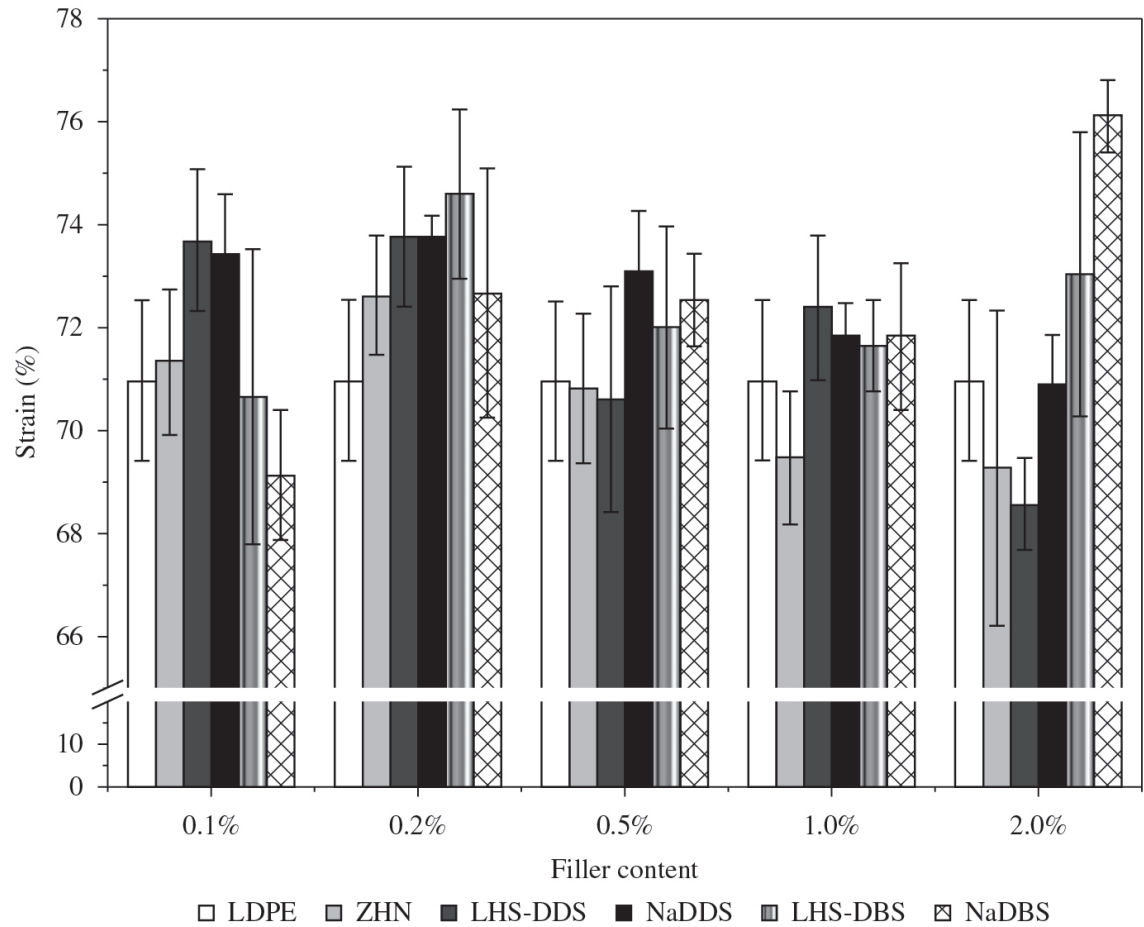

Figure 8. Strain at break of the LDPE composites using different fillers. 


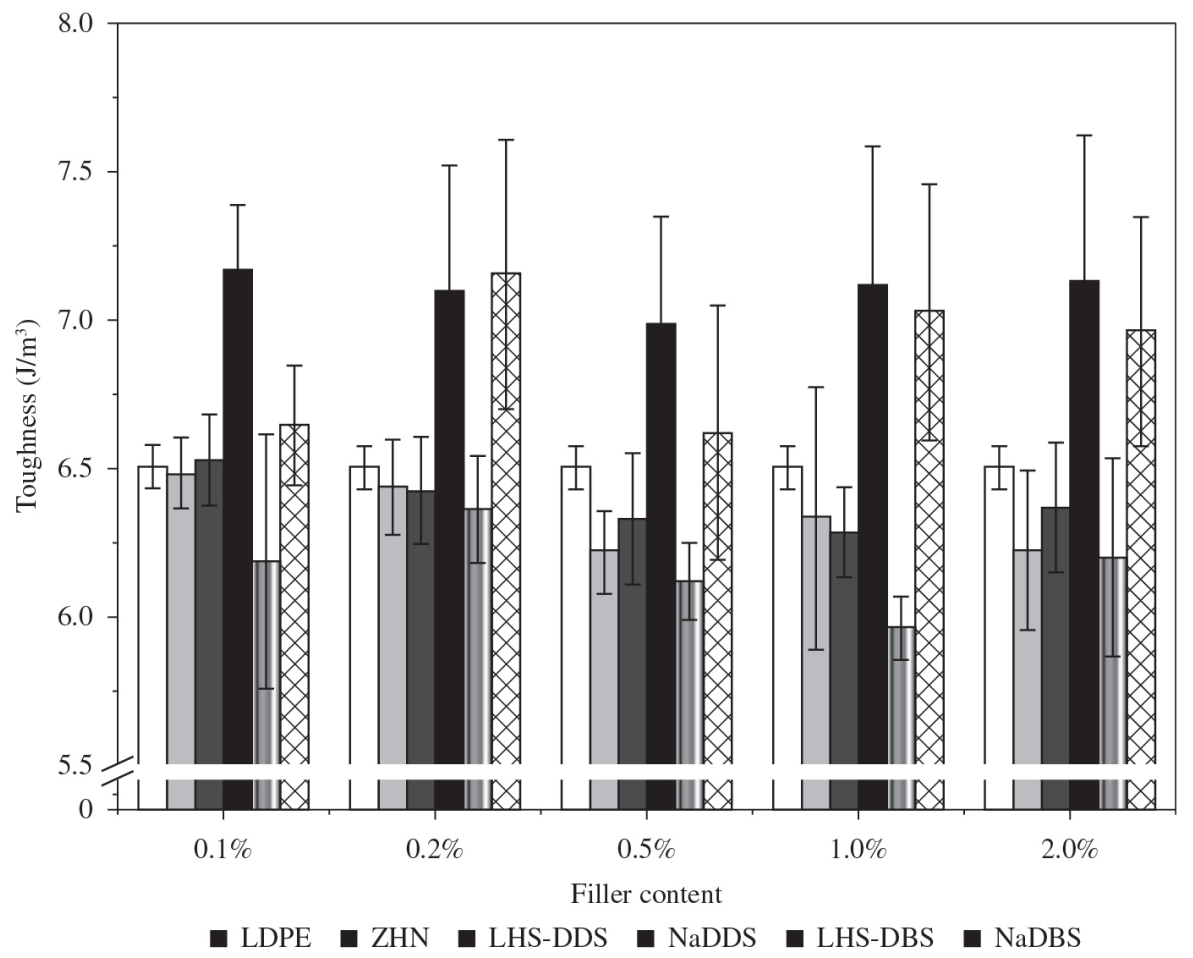

Figure 9. Toughness of the LDPE composites using different fillers.

for a more homogeneous and greater elongation of the polymer chains and their yield planes.

In addition, the composites with surfactants as fillers showed higher toughness (Figure 9). On the other hand, addition of DDS or DBS intercalated hydroxide salts decreased toughness in relation to the pure polymer. The yield of the polymer in a high-deformation situation occurs as a result of shear, which is also related to the interaction between polymer and filler.

\section{Conclusions}

Layered hydroxide salts intercalated with nitrate, dodecylsulfate (DDS) and dodecyl benzene sulfonate (DBS) anions were successfully synthesized by coprecipitation in slightly alkaline $\mathrm{pH}$. The X-ray data showed basal spacing of 9.66, 33.15 and $26.38 \AA$ for the intercalation of nitrate, DBS and DDS anions, respectively, which was ratified by the presence of specific bands in the FTIR spectra. Due to the difficult fitting of the anionic surfactant between the LHS layers, these samples showed low-crystallinity and small particle sizes. The interlayer distances were consistent with the intercalation of anionic species, connected to the tetrahedrally coordinated zinc, where water molecules are solvating the hydrophilic surfactant heads.

The results also confirm the possibility of preparing LDPE composites with homogeneously distributed hydrophilic (HZN) or hydrophobic (LHS-DDS e LHSDBS) fillers by simple melting compounding in an extruder. The fillers did not show a significant effect on either crystallization or melting of the polymer, but only a minor increase in melting and crystallization enthalpies, especially for lower filler content. The most interesting results regarding mechanical properties of the composites were obtained when using the layered fillers with surfactant anions. In addition, the composites with DDS intercalated hydroxide salts did not show significant variation in strength or toughness in comparison with the pure polymer, with only a minor increase in elongation at break. Mainly due to the small size of the particles, more prominent results were not obtained even though exfoliation of the hydrophobic compounds was confirmed by X-ray diffraction analyses.

In all, it can be concluded that fillers based on layered hydroxide salts may be interesting alternatives in the production of thermoplastic polymeric composites, especially those containing hydrophobic anions, such as those of the surfactants. Properties like flame retardancy and gas permeability should also be investigated since it is expected to significantly improve with the incorporation of this type of fillers ${ }^{[27,28]}$.

\section{Acknowledgements}

The authors would like to acknowledge the financial support from CAPES, Finep, CNPq, Nennan Project (Araucária Foundation/ $\mathrm{CNPq}$ ) and also to thank Braskem for polymer donation.

\section{References}

1. Dorigato, A. \& Pegoretti, A. - Eng. Fract. Mech., 79, p.213 (2012). http://dx.doi.org/10.1016/j.engfracmech.2011.10.014.

2. Coelho, P. H. D. L. \& Morales, A. R. - Polímeros, 23, p.410 (2013). 
3. Miranda, V. R. \& Carvalho, A. J. F. - Polímeros, 21, p.353 (2011). http://dx.doi.org/10.1590/S0104-14282011005000067.

4. Wang, L. J.; Xu, X. Y.; Evans, D. G. \& Li, D. Q. - Ind. Eng. Chem. Res., 49, p.5339 (2010). http://dx.doi.org/10.1021/ ie9016978.

5. Pires, M.; Petzhold, C. L.; Santos, R. V.; Perão, L. \& Chies, A. P. - Polímeros, 24, p.237 (2014).

6. Lu, Z.; He, C. \& Chung, T. S. - Polymer (Guildf.)., 42, p.5233 (2001). http://dx.doi.org/10.1016/S0032-3861(00)00806-5.

7. Peng, D.; Wei, C. \& Baojun, Q. - Prog. Nat. Sci., 16, p.573 (2006). http://dx.doi.org/10.1080/10020070612330037.

8. Arizaga, G. G. C.; Satyanarayana, K. G. \& Wypych, F. - Solid State Ion., 178, p.1143 (2007). http://dx.doi.org/10.1016/j. ssi.2007.04.016.

9. Marangoni, R.; Ramos, L. P. \& Wypych, F. - J. Colloid Interface Sci., 330, p.303 (2009). http://dx.doi.org/10.1016/j. jcis.2008.10.081. PMid:19081109

10. Marangoni, R.; Mikowski, A. \& Wypych, F. - J. Colloid Interface Sci., 351, p.384 (2010). http://dx.doi.org/10.1016/j. jcis.2010.08.006. PMid:20804981

11. Silva, M. L. N.; Marangoni, R.; Cursino, A. C. T.; Schreiner, W. H. \& Wypych, F. - Mater. Chem. Phys., 134, p.392 (2012). http://dx.doi.org/10.1016/j.matchemphys.2012.03.007.

12. Silva, M. L. N.; Marangoni, R.; Silva, A. H. \& Wypych, F. Polímeros, 23, p.248 (2013).

13. Zimmermann, A.; Jaerger, S.; Zawadzki, S. F. \& Wypych, F. - J. Polym. Res., 20, p.224 (2013).

14. Muksing, N.; Magaraphan, R.; Coiai, S. \& Passaglia, E. - Express Polym. Lett., 5, p.428 (2011). http://dx.doi. org/10.3144/expresspolymlett.2011.42.

15. Coiai, S.; Scatto, M.; Conzatti, L.; Azzurri, F.; Andreotti, L.; Salmini, E.; Stagnaro, P.; Zanolin, A.; Cicogna, F. \& Passaglia, E. - Polym. Adv. Technol., 22, p.2285 (2011). http://dx.doi. org/10.1002/pat.1759.

16. Ye, L. \& Wu, Q. H. - J. Applied Pol. Sci., 123, p.316 (2012). http://dx.doi.org/10.1002/app.33770.
17. Costa, F. R.; Wagenknecht, U. \& Heinrich, G. - Polym. Degrad. Stabil., 92, p.1813 (2007). http://dx.doi.org/10.1016/j. polymdegradstab.2007.07.009.

18. Arizaga, G. G. C.; Mangrich, A. S.; Gardolinski, J. E. F. C. \& Wypych, F. - J. Colloid Interface Sci., 320, p.168 (2008). http://dx.doi.org/10.1016/j.jcis.2007.12.038.

19. Stählin, W. \& Oswald, H. R. - J. Solid State Chem., 3, p.252 (1971). http://dx.doi.org/10.1016/0022-4596(71)90037-5.

20. Ardanuy, M. \& Velasco, J. I. - Appl. Clay Sci., 51, p.341 (2011). http://dx.doi.org/10.1016/j.clay.2010.12.024.

21. Majoni, S.; Su, S. \& Hossenlopp, J. M. - Polym. Degrad. Stabil., 95, p.1593 (2010). http://dx.doi.org/10.1016/j. polymdegradstab.2010.05.033.

22. Sun, Z.; Jin, L.; Shi, W.; Wei, M.; Evans, D.G. \& Duan, X. - Langmuir, 27, p.7113 (2011). http://dx.doi.org/10.1021/ la200846j.

23. Kandare, E.; Chigwada, G.; Wang, D. Y.; Wilkie, C. A. \& Hossenlopp, J. M. - Polym. Degrad. Stabil., 91, p.1781 (2006). http://dx.doi.org/10.1016/j.polymdegradstab.2005.11.021.

24. Biswick, T.; Jones, W.; Pacuła, A.; Serwicka, E. \& Podobinski, J. - J. Solid State Chem., 180, p.1171 (2007). http://dx.doi. org/10.1016/j.jssc.2007.01.012.

25. Morawiec, J.; Pawlak, A.; Slouf, M.; Galeski, A.; Piorkowska, E. \& Krasnikowa, N. - Eur. Polym. J., 41, p.1115 (2005). http:// dx.doi.org/10.1016/j.eurpolymj.2004.11.011.

26. Youssef, A. M.; Bujdosó, T.; Hornok, V.; Papp, S.; Hakim, A. F. A. \& Dékány, I. - Appl. Clay Sci., 77-78, p.46 (2013). http://dx.doi.org/10.1016/j.clay.2013.03.011.

27. Kiliaris, P. \& Papaspyrides, C. D. - Prog. Polym. Sci., 35, p. 902 (2010). http://dx.doi.org/10.1016/j.progpolymsci.2010.03.001.

28. Ye, L. \& Wu, Q. H. - J. Applied Pol. Sci., 123, p.316 (2012). http://dx.doi.org/10.1002/app.33770.

Received: Apr: 07, 2014 Accepted: June 26, 2014 